\title{
Geodesic Surfaces in Knot Complements
}

\author{
Iain R. Aitchison and J. Hyam Rubinstein
}

\section{CONTENTS}

1. Introduction

2. The Tessellation $\{5,3,6\}$ of Hyperbolic Space

3. The Dodecahedral Knots

4. $\Pi_{1}$-Injective Surfaces

5. Ideal Regular Dodecahedra in Hyperbolic Space

6. Tetrahedral Group Generators

7. Cusp and Trace Fields

8. Dihedral Angles

9. Another View of the Dodecahedron

10. Yet Another Description

11. Matrix Generators for the Tessellation $\{5,6\}$

12. Length Spectra

13. Open Problems

Acknowledgements

References
We construct closed, embedded, totally geodesic surfaces in two hyperbolic manifolds obtained as knot complements.

\section{INTRODUCTION}

There is considerable interest in the question of existence of $\pi_{1}$-injective closed surfaces, immersed or embedded, in three-manifolds. If a three-manifold is endowed with a metric, we may ask whether such a surface exists which is totally geodesic with respect to the given metric.

In the case of hyperbolic three-manifolds, Long [1987] has shown that if such immersed surfaces exist there is a finite cover containing an embedded totally geodesic closed surface. Menasco and Reid [1992] have obtained results on the nonexistence of embedded totally geodesic closed surfaces in certain link complements in the three-sphere.

For link complements admitting an arithmetic structure, examples of closed immersed totally geodesic surfaces are fairly easy to construct when the link has more than one component. At a more algebraic level, Maclachlin [1986] has proved the existence of Fuchsian subgroups of arithmetic groups $\operatorname{PSL}\left(2, \mathcal{O}_{d}\right)$, but without describing their realizations as compact or noncompact surfaces in threemanifolds.

Alan Reid [1991b] has shown by arithmetic arguments that the complement of the figure-eight knot in $S^{3}$, with its complete hyperbolic metric, contains infinitely many commensurability classes of totally geodesic immersed closed surfaces. Commensurability classes in the arithmetic case are also discussed in [Maclachlan and Reid 1987]. The figureeight knot is the only arithmetic knot, a result also due to Reid [1991a]. No other hyperbolic knot 
complements were known to contain immersed totally geodesic closed surfaces. No explicit description of those in the figure-eight knot complement have appeared in the literature.

We describe two knots containing such surfaces, the dodecahedral knots $D_{f}$ and $D_{s}$. The knot $D_{f}$ was constructed by Thurston in 1982 [Riley 1989]. We describe an orientation-reversing involution on the complement of Thurston's knot $D_{f}$, as well as one on $D_{s}$. Our approach derives partly from conversations with Bill Thurston and is constructive. The knot $\mathcal{D}_{s}$ should be named "Long's knot", since its construction derives from the question "Is there...?", asked of the authors by Long.

This paper is organized as follows. We start by giving a nonanalytic direct argument for the existence of closed totally geodesic immersed surfaces in these knot complements, and recall the knots' original construction. Two pictures of the view from infinity of an ideal dodecahedron are given, the first with a vertex at infinity. This enables us to see directly the cusp and trace fields described in [Neumann and Reid 1992], but is also an excuse to draw some beautiful pictures related to stellated Platonic solids and the face structure of icosahedra. We calculate the dihedral angles for the immersed surfaces, and matrix representatives for the tessellation $\{5,6\}$ of the immersed surface. The second view, again in the upper-half space model for hyperbolic space, has an endpoint of a fivefold axis of symmetry at $\infty$, and derives metrically from two nested pentagrams associated to an immersed nonregular decagon. Finally we present the initial segments of the length spectra of these knots, without offering any explanation for their surprising similarity.

This work first appeared as a University of Melbourne Research Report in 1990. Our motivation was the belief that a high degree of symmetry of the tessellation of the universal cover of a hyperbolic knot complement might yield an equivariant collection of hyperbolic planes giving a closed orientable immersed totally geodesic surface in the knot complement. Since the two dodecahedral knots arise from the same tessellation, it is natural to compare other geometric properties potentially shared by them, such as degree of symmetry, and length spectra. Amusingly, both knot complements admit nontrivial symmetries, whereas a motivation for Riley's examination of the complement of the knot $9_{32}$ was a claim made by Thurston in 1981 that the knot $D_{f}$ admitted no such nontrivial symmetry.

\section{THE TESSELLATION $\{5 ; 3 ; 6\}$ OF HYPERBOLIC SPACE}

Consider the unit-ball model for $\mathbb{H}^{3}$, with hyperbolic space modelled by the interior of the unit ball in $\mathbb{R}^{3}$. We use the standard Euclidean coordinates $x, y$ and $z$, with the $z$-axis vertical.

Take a Euclidean dodecahedron with two faces parallel to the $x y$-plane, with vertices lying on 4 meridians of the unit sphere, also parallel to the $x y$ plane. The $z$-axis is an axis for a fivefold rotational symmetry of the dodecahedron.

Keeping the same vertices, convert this Euclidean dodecahedron to an ideal hyperbolic dodecahedron $\mathcal{D}$. The dihedral angles become $\pi / 3$, and $\mathbb{H}^{3}$ can be tessellated by isometric copies of $\mathcal{D}$ by reflections in faces. This gives the tessellation $\{5,3,6\}$, with 6 dodecahedra about each edge [Coxeter 1956]. Note that each edge is a geodesic axis of sixfold rotational symmetry of the tessellation. Denote by $\Gamma$ the infinite group of symmetries of this tessellation. $\Gamma$ is generated by the 120 symmetries of $\mathcal{D}$, together with reflections in the faces of $\mathcal{D}$.

The $x y$-plane intersects $\mathcal{D}$ in a decagon. If we translate $\mathcal{D}$ upwards along the $z$-axis, we can arrange for $\mathcal{D}$ to have its bottom face $\mathcal{F}$ lying in the $x y$-plane as a regular ideal pentagon. The vertices of $\mathcal{D}$ still lie on horizontal meridians. At this stage, the $x y$-plane is tessellated by faces of dodecahedra, which thus induce the 2-dimensional hyperbolic tessellation $\{5, \infty\}$ [Coxeter 1956].

Choose one of the 5 edges of $\mathcal{D}$, designated $\mathcal{E}$, emanating from a vertex $\mathcal{V}$ of $\mathcal{F}$, with $\mathcal{E}$ not an edge of $\mathcal{F}$. The other endpoint of $\mathcal{E}$ lies on a meridian 
with positive $z$-coordinate, and consequently $\mathcal{E}$ is disjoint from the $z$-axis, but coplanar with it.

Slide $\mathcal{D}$ back down the $z$-axis until the edge $\mathcal{E}$ lies symmetrically about the $x y$-plane, and is therefore orthogonal to it. At this stage, the $x y$-plane is orthogonal to two distinct axes of rotational symmetry of the tessellation. Hence rotating about either the $z$-axis, or $\mathcal{E}$, both the tessellation and the $x y$-plane are preserved. The intersection of the $x y$-plane with $\mathcal{D}$ is now a regular $\pi / 3$-angled pentagon, since the plane is orthogonal to $\mathcal{E}$ and the other four edges emanating from the vertices of $\mathcal{F}$. Hence the intersections of the $x y$-plane with all dodecahedra of the tessellation $\{5,3,6\}$ induce the tessellation $\{5,6\}$ on the plane [Coxeter 1956]. The circle on the sphere at infinity corresponding to this plane is therefore disjoint from all ideal vertices of the tessellation $\{5,3,6\}$.

By symmetry, there is an infinite $\Gamma$-invariant family of planes in $\mathbb{H}^{3}$, the intersection each of which with any dodecahedron being either empty or a regular pentagon parallel to a unique face. Each dodecahedron contains 12 such pentagons of intersection. As an immediate consequence, we have the result:

Theorem 2.1. Suppose that $G$ is a torsion-free subgroup of $\Gamma$ of finite index. Then the finite-volume noncompact hyperbolic three-manifold $M_{G}=\mathbb{H}^{3} / G$ contains an immersed, totally geodesic closed surface.

Corollary 2.2. Each of the dodecahedral knots $D_{f}$, $D_{s}$ constructed in [Aitchison and Rubinstein 1992] contains an immersed closed surface in its complement in $S^{3}$, totally geodesic with respect to the complete constant curvature metric.

Proof. These knots are shown in [Aitchison and Rubinstein 1992] to arise as in the theorem.

\section{THE DODECAHEDRAL KNOTS}

We recall the construction of the two dodecahedral knots $D_{s}$ and $D_{f}$ [Aitchison and Rubinstein 1992]. Take two copies of an ideal regular hyper- bolic dodecahedron, and colour the faces of one using two colours, so that no vertex is surrounded by faces all of the same colour. Identify corresponding faces of the two copies by isometries, rotations of $\pm 2 \pi / 5$, with sign determined by the colour of the face. There are essentially two choices for such colourings, as shown in Figure 1, and the resulting topological spaces after face identifications are the complements of the two knots $D_{s}$ and $D_{f}$. The knots $D_{s}$ and $D_{f}$ are illustrated in Figure 2 .
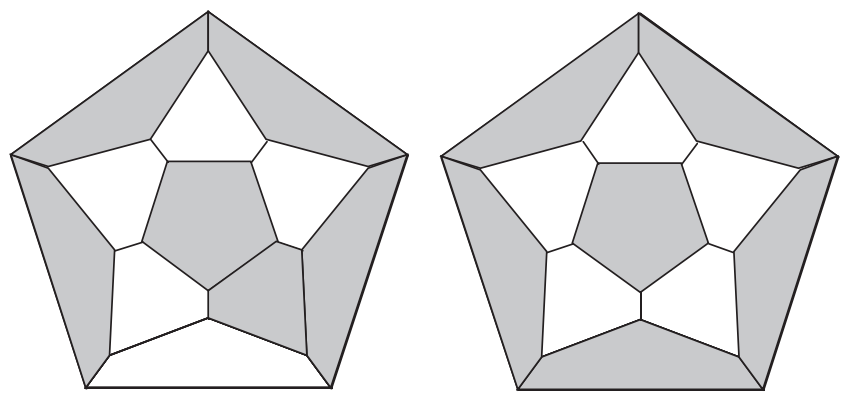

FIGURE 1. The two ways to shade the faces of a dodecahedron with two colors so that no vertex is surrounded by faces all of the same color.

Proposition 3.1. The two knots $D_{s}$ and $D_{f}$ are distinct.

Proof. The Seifert surfaces determined by applying Seifert's algorithm to the given projections are minimal [Murasugi 1963], and of different genus. The knot $D_{f}$ is fibred [Aitchison and Rubinstein 1992], with fibre surface that from the projection, and hence is distinct from $D_{s}$, which has lower genus.

Neumann and Reid [1992] have demonstrated some remarkable properties of these knots:

(i) These are the first known examples of knots with cusp field and trace field differing (respectively $\mathbb{Q}(\sqrt{-3})$ and $\mathbb{Q}(\sqrt{-3}, \sqrt{5})$.

(ii) They are the only known nonarithmetic knots with cusp field $\mathbb{Q}(\sqrt{-3})$, that of the figure-eight knot.

(iii) They are the only known nonarithmetic knots with hidden symmetries. (Equivalently, the knot complements nonnormally cover some orbifold.) 


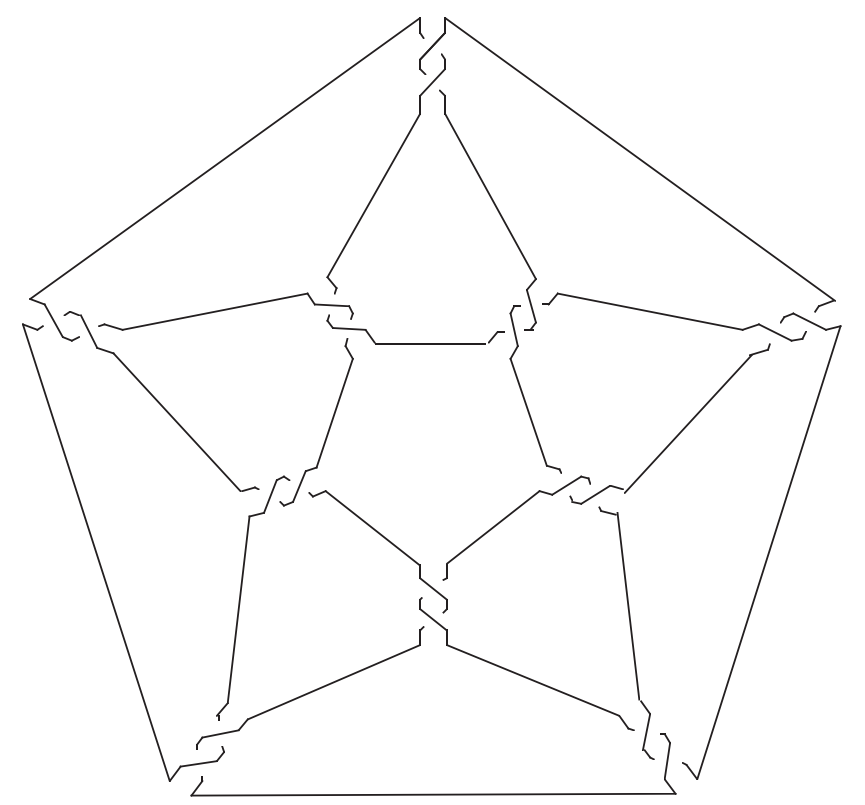

FIGURE 2. The knots $D_{s}$ (left) and $D_{f}$ (right).

\section{4. $\Pi_{1}$-INJECTIVE SURFACES}

In [Aitchison and Rubinstein 1992], it is shown that these knot complements admit singular polyhedral metrics, with respect to which there exist immersed $\pi_{1}$-injective totally geodesic closed surfaces $\mathcal{S}_{s}, \mathcal{S}_{f}$. Each of these surfaces arises topologically by identifying edges of the 24 pentagons parallel to the 24 faces of the two dodecahedra. The surfaces so obtained are topologically exactly those appearing in the smooth construction.

Theorem 4.1. The surfaces $\mathcal{S}_{s}$ and $\mathcal{S}_{f}$ are regularly homotopic to totally geodesic closed surfaces in the standard complete hyperbolic metric on $S^{3}-D_{s}$ and $S^{3}-D_{f}$ respectively.

\section{IDEAL REGULAR DODECAHEDRA IN HYPERBOLIC SPACE}

Coxeter [1948; 1949] described the four possible tessellations of $\mathbb{H}^{3}$ by regular ideal Platonic solids. The tessellation $\{5,3,6\}$, corresponding to the regular ideal dodecahedron $\mathcal{D}$, has symmetry group

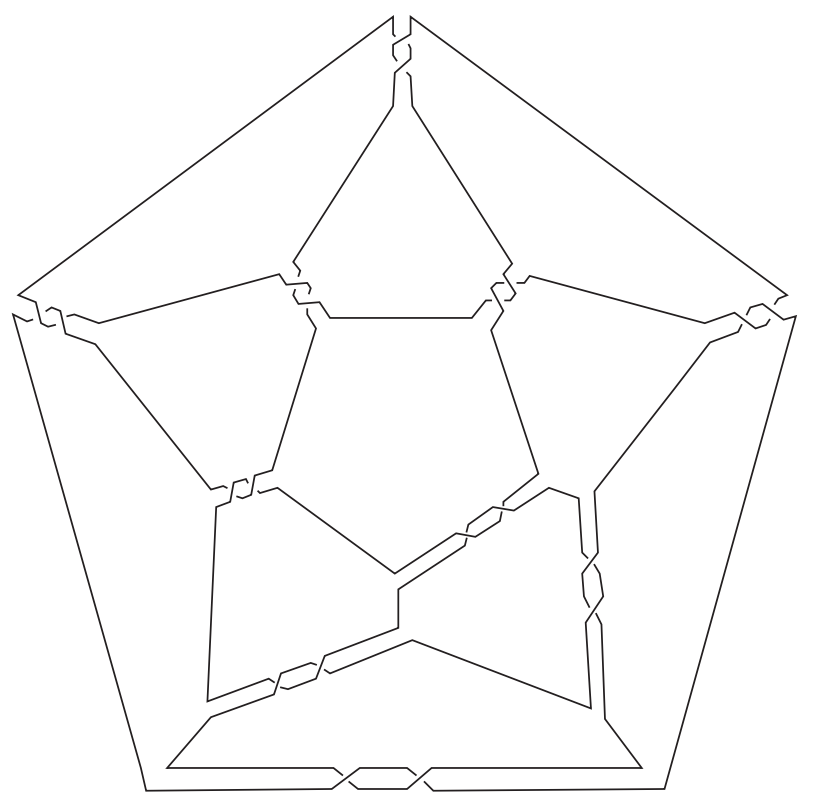

$\Gamma$ the tetrahedral group $T[5,2,2 ; 2,3,6]$ [Neumann and Reid 1992; Lee 1985a]. This is generated by rotations in the edges of one of the 120 isometric tetrahedra of the barycentric subdivision of $\mathcal{D}$. Thus $\Gamma$ is also generated by reflections in the faces of $\mathcal{D}$, together with the order-120 group of symmetries of $\mathcal{D}$. There are six dodecahedra around each edge, so the dihedral angles are all $\pi / 3$.

We determine matrices in $\mathrm{SL}(2 ; C)$ corresponding to generators of $\Gamma$. The tetrahedron has vertices $v, e, f, c$, with $v$ at infinity, and with $e, f$ and $c$ corresponding to an edge, a face and the centre of $\mathcal{D}$ respectively.

We use the upper-half space model for $\mathbb{H}^{3}$, with the ideal vertex $v$ of the tetrahedron at $\infty$. The geodesic edges $e v, f v$ and $c v$ become parallel vertical lines in this model, with dihedral angles $\pi / 6$, $\pi / 2$ and $\pi / 3$ respectively. Viewed from $\infty$, as in Figure 3, the vertices $e, f$ and $c$ project to the vertices of the triangle $E, F$ and $C$ arranged to be at the points $0,-\tau / 2$ and $\tau \xi^{5} / \sqrt{3}$ where $\xi=$ $\exp i \pi / 6=\sqrt{3} / 2+i / 2$ in the complex plane, and $\tau=(1+\sqrt{5}) / 2$ is the golden mean. 


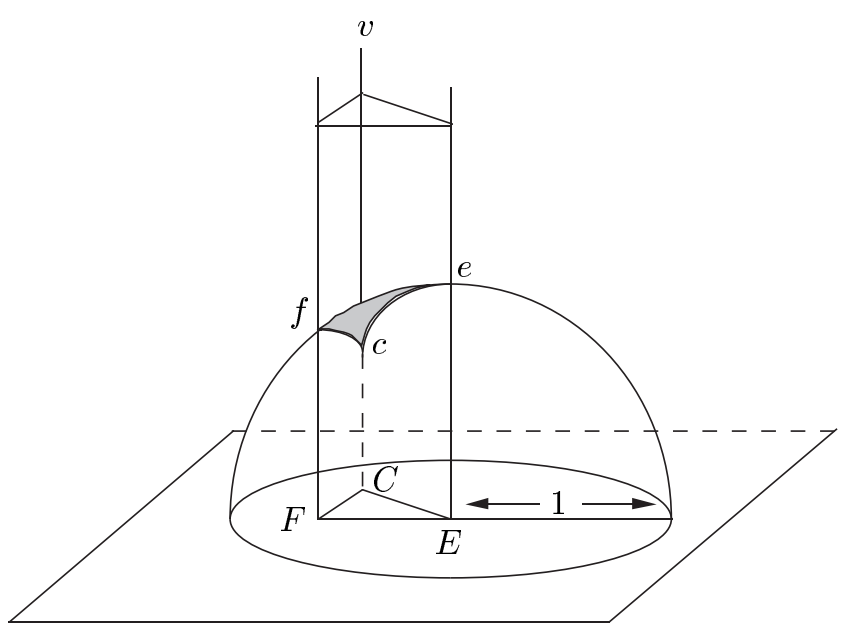

FIGURE 3. Elements of the hyperbolic tetrahedron $\mathcal{D}$, with vertices $c, e, f$, and $v$ (at infinity).

The face ecf of the tetrahedron is the intersection of the triangular chimney over ECF with the hyperbolic plane corresponding to the circle in the complex plane of radius 1 , centred at $E=0$. It is a straightforward calculation to show that the dihedral angles at the edges $e c, c f$ and $f e$ are respectively $\pi / 2, \pi / 5$ and $\pi / 2$. This completes the construction of the tetrahedron $[5,2,2 ; 6,3,2]$. See Figure 3.

\section{TETRAHEDRAL GROUP GENERATORS}

The tetrahedral group $T\left[\lambda_{1}, \lambda_{2}, \lambda_{3} ; \mu_{1}, \mu_{2}, \mu_{3}\right]$ determined by positive integers $\lambda_{1}, \lambda_{2}, \lambda_{3}, \mu_{1}, \mu_{2}, \mu_{3}$ has presentation [Lee 1985b]

$$
\begin{aligned}
& \langle x, y, z: \\
& \left.\quad x^{\lambda_{1}}=y^{\lambda_{2}}=z^{\lambda_{3}}=(x y)^{\mu_{1}}=(x z)^{\mu_{2}}=\left(y^{-1} z\right)^{\mu_{3}}=1\right\rangle .
\end{aligned}
$$

In this notation, we have $T[5,2,2 ; 6,3,2]$ generated by these Möbius transformations:

rotation about $c f$ by $2 \pi / 5=x=\left(\begin{array}{cc}-\tau & -1 \\ 1 & 0\end{array}\right)$ rotation about $f e$ by $2 \pi / 2=y=\left(\begin{array}{cc}0 & i \\ i & 0\end{array}\right)$

rotation about $e c$ by $2 \pi / 2=z=\left(\begin{array}{cc}0 & i \xi^{-1} \\ i \xi & 0\end{array}\right)$ rotation about $e v$ by $2 \pi / 6=y^{-1} z=\left(\begin{array}{cc}\xi & 0 \\ 0 & \xi^{-1}\end{array}\right)$ rotation about $c v$ by $2 \pi / 3=x z=\left(\begin{array}{cc}-i \xi & -i \tau \xi^{-1} \\ 0 & i \xi^{-1}\end{array}\right)$ rotation about $f v$ by $2 \pi / 2=x y=\left(\begin{array}{cc}-i & -i \tau \\ 0 & i\end{array}\right)$

The fixed points on the sphere at $\infty$ corresponding to these elliptic elements are $\infty, E, C$ and $F$, corresponding to the latter 3 elements, and the intersections of the unit circle with the straight lines determined by $C F, F E$ and $E C$. From the dodecahedral symmetry, we know that the hyperbolic plane in $\mathbb{H}^{3}$ determined by ecf contains two edges, meets another two edges orthogonally, bisects four faces and contains four ideal vertices. Similarly the endpoints of the geodesic determined by $v c$ are both ideal vertices.

\section{CUSP AND TRACE FIELDS}

We can obtain a view of the cusp structure by a slight elaboration of Figure 3, enabling the cusp and trace fields to be seen directly. Coxeter [1948; 1949] does not treat stereographic projection, so we give a fairly complete description.

In Figure 4, left, we have constructed an equilateral triangle $E, P, Q$ of base length $\tau$. The circle of radius 1 centred at $E=0$ intersects the edges of this triangle at the points $R_{E}$ and $S_{E}$. Similarly, unit circles centred at $P$ and $Q$ determine the points $R_{P}, S_{P}, R_{Q}$ and $S_{Q}$. Next we add the edges joining vertices of the equilateral triangle to the two points just constructed on the opposite edge. These segments intersect in twelve points, six of which are labelled $A_{1}, \ldots, A_{6}$, and three $B_{1}, B_{2}, B_{3}$ in the figure. Points $A_{6}$ and $A_{3}$ are, like $S_{E}$ and $R_{E}$, at distance 1 from $E$. We draw segments joining the $B_{i}$ to the center $C$ of the original triangle, and the segments $A_{1} A_{6}, A_{2} A_{3}$, and $A_{4} A_{5}$.

Erasing superfluous lines (Figure 4, right) we are left with a pattern of nine pentagons:

$$
Q R_{E} A_{3} A_{2} S_{P}, E R_{P} A_{5} A_{4} S_{Q}, P R_{Q} A_{1} A_{6} S
$$


at the vertices of the original triangle;

$$
R S_{Q} A_{4} B_{2} A_{3}, R_{P} S A_{6} B_{3} A_{5}, R_{Q} S_{P} A_{2} B_{1} A_{1}
$$

at the edges; and

$$
A_{2} A_{3} B_{2} C B_{1}, A_{4} A_{5} B_{3} C B_{2}, A_{6} A_{1} B_{1} C B_{3}
$$

at the centre.

Together with the three pentagons $\infty P R_{Q} S_{P} Q$, $\infty Q R S_{Q} E$ and $\infty E R_{P} S P$, we have the twelve faces of the ideal dodecahedron $\mathcal{D}$.

Viewed from the parabolic fixed point at infinity, we see the tessellation of the plane by equilateral triangles, each of which corresponds to an ideal dodecahedron with a vertex at $\infty$. We see directly that the cusp field is $\mathbb{Q}(\sqrt{-3})$. Moreover, from [Neumann and Reid 1992] we know that the trace field is generated by parabolic fixed points in the plane, after three of which have been conjugated to lie at the points $0, \pm 1, \infty$. From the matrix generators of the symmetry group $\Gamma$, and the existence of a parabolic fixed point at $\pm \tau$, we conclude as

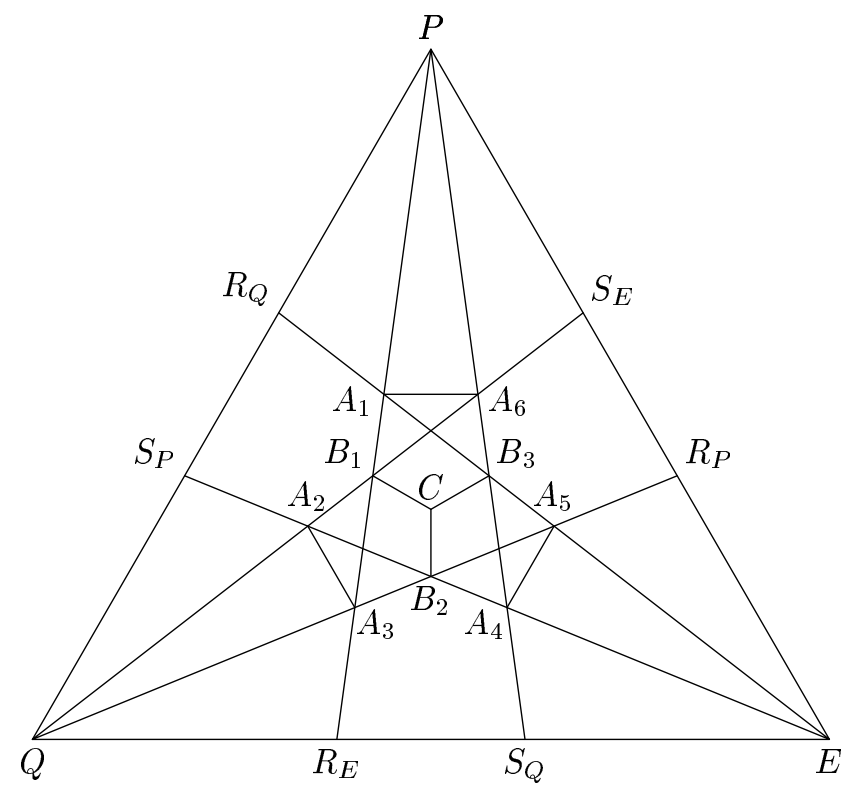

in [Neumann and Reid 1992] that the trace field is $\mathbb{Q}(\sqrt{-3}, \sqrt{5})$.

The reader may have observed the similiarity between Figure 4 and the diagram appearing in [Coxeter 1956, p. 97], showing the 18 lines of intersection of planes determined by faces of a regular Euclidean icosahedron with the plane of a distinguished face. These 18 lines are depicted in Figure 5. The three lines of the equilateral triangle, together with the remaining six lines through the vertices so determined, give nine lines from which the dodecahedron is constructed.

That this diagram arises can be understood as follows. Consider a spherical dodecahedron, and take the metric on the graph of vertices and edges, with all edges of length 1 . Choose any vertex $v$, and consider the circle $C_{v}$ passing through the six vertices at distance 2 from $v$. This circle lies in a plane orthogonal to the axis $v v^{*}$, where $v^{*}$ is the vertex antipodal to $v$. Dually, the six circles about the vertices at distance 2 from $v$, all pass through $v$. Another three planes passing through $v$ are de-

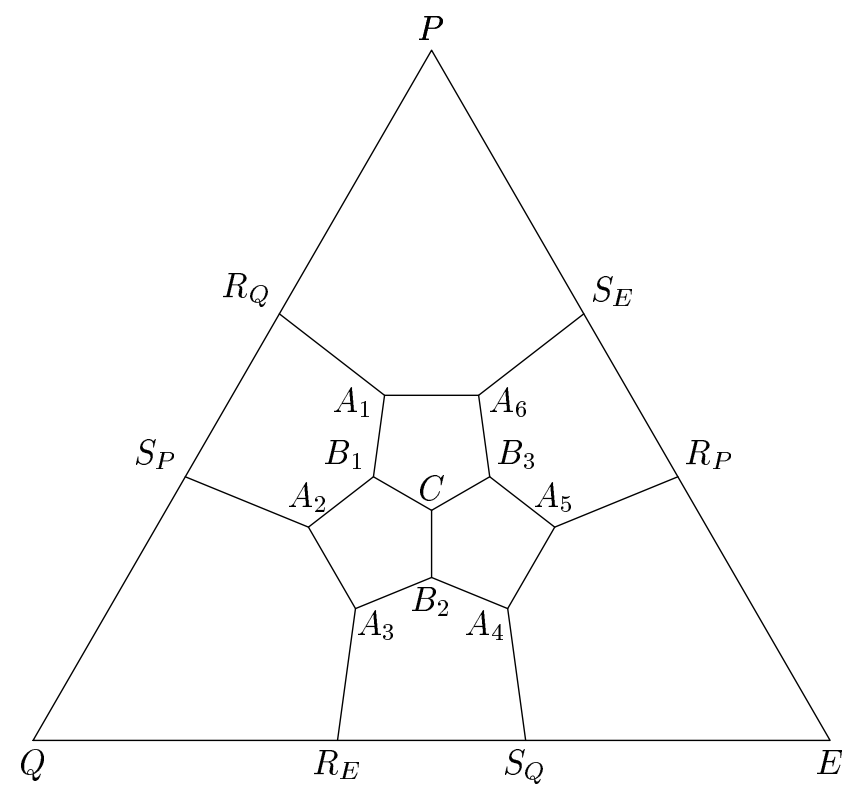

FIGURE 4. Left: Triangle $E P Q$ has side length $\tau=(1+\sqrt{5}) / 2$. On the edges we mark $S_{E}$ and $R_{E}$ at distance 1 from $E$, etc. Connecting these points to the opposite vertices creates nine additional points $A_{1}, \ldots, A_{6}, B_{1}, \ldots, B_{3}$. Together with the center of the construction, all these points form 19 of the vertices of a dodecahedron, seen in stereographic projection from the last vertex (right). 


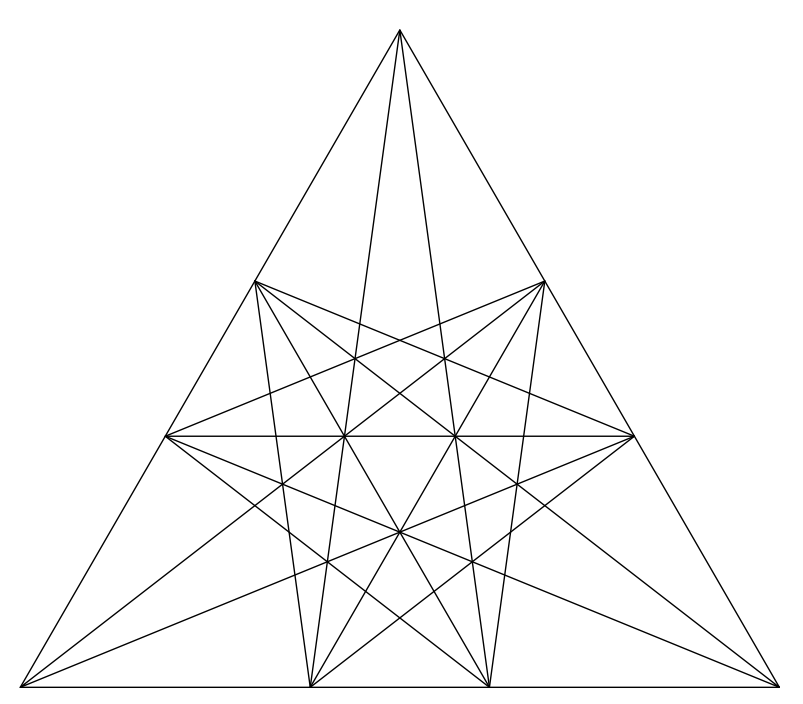

FIGURE 5. The 18 lines of intersection of planes determined by faces of a regular Euclidean icosahedron with the plane of a distinguished face.

termined by the three faces of the dodecahedron incident at $v$. Stereographically projecting the dodecahedron onto a plane from the point $v$, the 3 faces determine the equilateral triangle, and the six circles give rise to the six lines throught the vertices of the equilateral triangle. These six lines intersect in points corresponding to the vertices of the dodecahedron at which the circles intersect.

The icosahedron can be constructed by truncating the vertices of a dodecahedron by planes orthogonal to radial lines from the centre of the dodecahedron to the vertices. The six lines above thus correspond to six faces of the icosahedron.

The existence of these six circles through each vertex of the dodecahedron facilitates the construction of the diagram directly. The regular Euclidean or spherical dodecahedron, stereographically projected from a vertex onto the tangent plane of the sphere at the antipodal vertex, appears as in Figure 4 , right, since there are straight lines containing six vertices, such as $Q A_{3} B_{2} A_{5} R_{P} \infty$. Calculating the tangent of the angle $R_{P} Q E$ in different ways leads to a simple quadratic equation whose solution involves the golden mean.

\section{DIHEDRAL ANGLES}

We have given a geometric argument for the existence of the family of hyperbolic planes in section 2 , using the ball model for hyperbolic space. In this section we consider some analytic aspects, in order to calculate the dihedral angle between any two such planes.

We have associated a hypersurface $\mathcal{H}\left(\mathcal{D}, \mathcal{F}, \mathcal{E}_{i}\right)$ to each triple $\left(\mathcal{D}, \mathcal{F}, \mathcal{E}_{i}\right)$, where $\mathcal{D} \supset \mathcal{F}$ and $\mathcal{E}_{i}$ has an ideal vertex in common with $\mathcal{F}$. By rotational symmetry about the fivefold axis $\gamma_{F}$ orthogonal to $\mathcal{F}$, this hypersurface is independent of edge choice $\mathcal{E}_{i}$.

Now consider the family $\mathcal{H}_{\Gamma}$ of all hypersurfaces arising in this fashion. We use the view from infinity to calculate the dihedral angle between any two intersecting planes in $\mathcal{H}_{\Gamma}$. Without loss of generality we may consider the following situation.

Denote by $\gamma$ the geodesic axis of fivefold rotational symmetry preserving the ideal pentagonal face $\mathcal{F}=A_{3} B_{2} A_{4} S_{Q} R_{E}$ of the dodecahedron $\mathcal{D}$ of Figure 4. Thus $\gamma$ also passes through the centre of the face $P R_{Q} A_{1} A_{6} S_{E}$. The hyperbolic plane $\mathcal{H}(\mathcal{D}, \mathcal{F})$ is orthogonal to the edges $Q R_{E}, A_{3} A_{2}$, $B_{2} C, A_{4} A_{5}, S_{Q} E$, as well as to the adjacent faces to $\mathcal{F}$. Hence $\mathcal{H}(\mathcal{F})=\mathcal{H}(\mathcal{D}, \mathcal{F})$ has $F$ as its centre for the corresponding limit circle $C_{1}$ at infinity, by symmetry. This circle meets the (planar) edges just mentioned orthogonally, and inverting in the circle interchanges the endpoints of these edges. This explains why the edges, extended into the interiors of pentagons, are concurrent. In particular, we see another reason why the centre of $C_{1}$ is the point $F$.

To calculate the radius $\rho_{1}$ of $C_{1}$, change coordinates so that $F$ is the origin, and $Q, E$ lie at the points $\pm \tau / 2$ on the $x$-axis. Then $S_{Q}$ and $R_{E}$ are respectively $\pm(2-\tau) / 2$. Hence

$$
\rho_{1}^{2}=\frac{(2-\tau)}{2} \cdot \frac{\tau}{2}=\frac{(\tau-1)}{4}
$$

Another hyperbolic plane in $\mathcal{H}_{\Gamma}$ is orthogonal to the sixfold axis $e v$, and thus has its circle at infinity 
$C_{2}$ centred at $E$, and orthogonal to the edges $E \infty$, $R_{P} S_{E}, A_{5} B_{3}, A_{4} B_{2} ; S_{Q} R_{E}$. We calculate its radius $\rho_{2}$ analogously, since inversion in $C_{2}$ interchanges $R_{E}$ and $S_{Q}$.

$$
\rho_{2}^{2}=|1-\tau| .1=(\tau-1) .
$$

The angle at which the circles $C_{1}$ and $C_{2}$ intersect in the plane is exactly the dihedral angle at which the corresponding planes in hyperbolic space meet. Since the centres of these circles are at distance $\tau / 2$ apart, we calculate this angle as

$$
\begin{aligned}
\cos \theta & =\frac{\rho_{1}^{2}+\rho_{2}^{2}-\tau^{2} / 4}{2 \rho_{1} \rho_{2}}=(2 \tau-3) \frac{\tau}{2}=1-\frac{\tau}{2} \\
& =\frac{3-\sqrt{5}}{4} \approx 0.190983006
\end{aligned}
$$

so $\theta \approx 79^{\circ}$.

Remark 8.1. Hass and Scott [1992] have shown that immersed surfaces meeting at such an angle automatically satisfy their 4-plane, 1-line property.

We have drawn circles determining such hypersurfaces in Figure 6. Note that there are six pentagons sliced off by the hypersurface orthogonal to the sixfold $z$-axis.

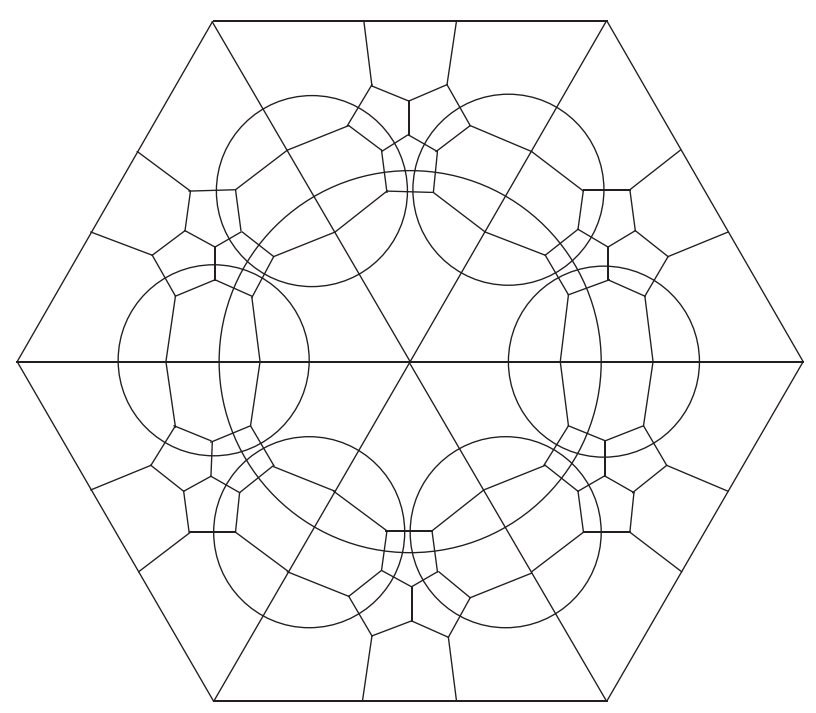

FIGURE 6. The circle $C_{2}$ and six copies of $C_{1}$, on the sphere at infinity (as seen from one point thereof).

\section{ANOTHER VIEW OF THE DODECAHEDRON}

That the surfaces constructed above slice off pentagons parallel to the faces of dodecahedra can also be seen by a different view of the dodecahedron, again in the upper-half plane model, but conjugated so that the endpoints of an axis of fivefold rotation lie at 0 and $\infty$. We will eventually describe a simple construction of the stereographic projection of a regular dodecahedron based on an irregular decagon.

We begin with a ruler and compass construction (Figure 7), and then reconcile the emergent picture with the tessellation using cross-ratios. We start by constructing two lines through an initial point $O$, at an angle of $\pi / 5$ (recall that the regular pentagon is constructible!). Choose a point $A$ on one of these lines, and construct $B$ as the intersection of the other line with the line $A B$ at an angle of $\pi / 3$ to $O A$. Similarly construct $C$ on the line $O A$ using a line at angle $\pi / 3$ to $A B$ at $B$. With a compass centred at $B$, construct the isosceles triangle $C B D$, with $B C=B D$. Construct a line through $D$ at angle $\pi / 3$ to $O B$ at $D$, thereby finding $E$ on $O A$. Finally construct $F$ on $O B$, so that $\angle D E F=\pi / 3$.

Choose orthogonal axes through $O$ and a scale so that $F$ is the point 1 in the complex plane. The point $D$ is thus at the point $w$ satisfying the quadratic equation

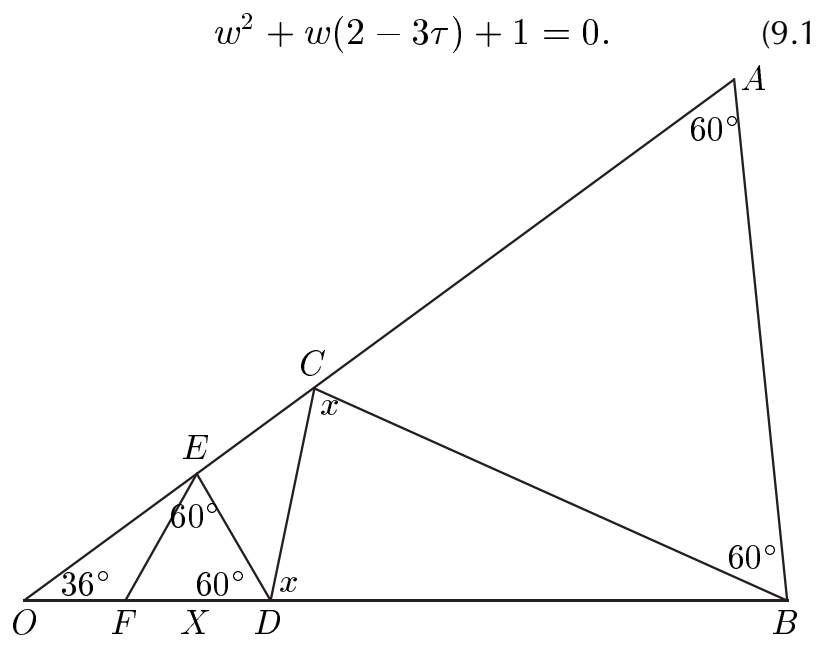

FIGURE 7. A ruler and compass construction. 
This can be obtained by dropping a perpendicular from $E$ to $X$ on $O B$, and calculating $\cos \pi / 5=$ $O X / O E:$ if $|O X|=z$, and $|O D|=w$, then

$$
\begin{aligned}
|O X| & =(z+w) / 2, \\
|E X| & =\sqrt{3}|F X|=\sqrt{3}(w-z) / 2 \\
|O E|^{2} & =|O X|^{2}+|E X|^{2} \\
\tau & =2 \cos \pi / 5=|E X| /|O E| .
\end{aligned}
$$

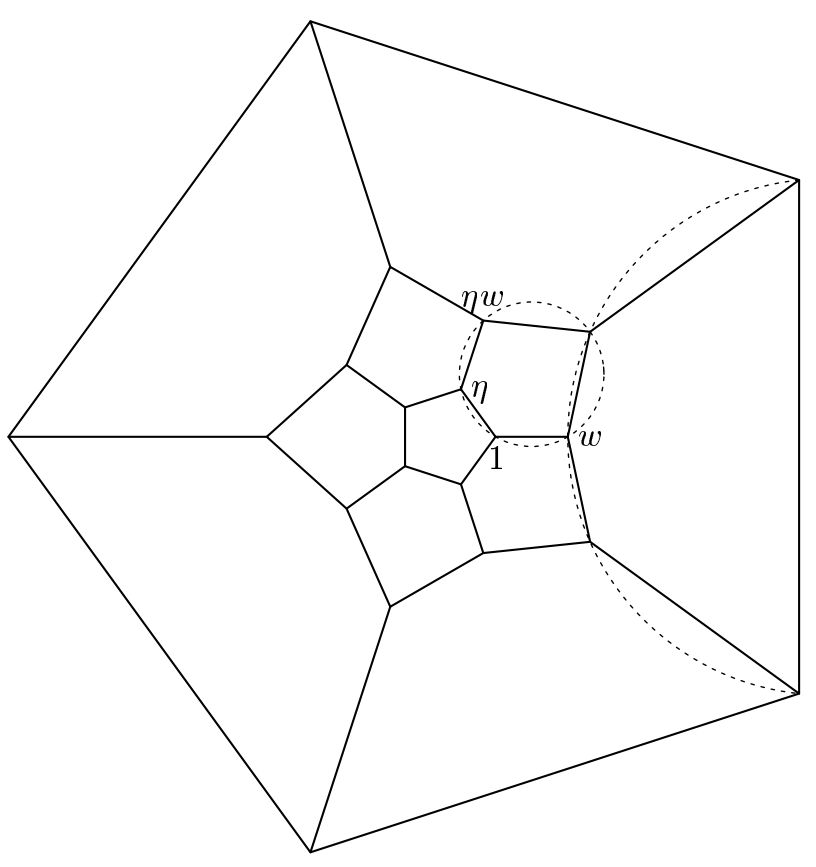

FIGURE 8. The points $F, D, C, A$ of Figure 7, plus their images under five-fold rotation about $O$, correspond to the vertices of an ideal regular hyperbolic dodecahedron $\mathcal{D}$.

Now take the four points $F, D, C, A$ and their images under fivefold rotation about $O$. This gives us the twenty points in Figure 8. It turns out that these twenty points are the vertices of a regular ideal dodecahedron in the upper half-space model, with fivefold axis of symmetry having endpoints at 0 and $\infty$.

To see this, note that, by symmetry considerations, the distribution of points of an ideal regular dodecahedron $\mathcal{D}$ in the required arrangement must be similar to that of Figure 8. It suffices to verify that Figure 8 is metrically correct.
Each face of $\mathcal{D}$ is a regular pentagon, so each four consecutive vertices on any face must have the same cross-ratio as corresponding points of the standard Euclidean regular pentagon. (Recall that the cross-ratio of four ordered points $z_{1}, z_{2}, z_{3}, z_{4}$ in the Riemann sphere is

$$
\left[z_{1}, z_{2}, z_{3}, z_{4}\right]=\frac{z_{1}-z_{3}}{z_{1}-z_{4}} \frac{z_{2}-z_{4}}{z_{2}-z_{3}}
$$

and that the cross-ratio is preserved under Möbius transformations.) Setting $\eta=\exp 2 \pi i / 5$, one can easily verify that the cross-ratio $\left[1, \eta, \eta^{2}, \eta^{3}\right]$ of four consecutive vertices of a regular pentagon, taken counter-clockwise, equals $\tau$. Also by direct computation, we see that the cross-ratio

$$
t=[\eta w, \eta, 1, w]
$$

of the four labeled points in Figure 8 satisfies the linear equation

$$
w^{2}+w(2-3 t)+1=0
$$

comparing with (9.1), we see that $t=\tau$.

To verify that the other points are correctly distributed, observe that, by construction, there are circles (whose circles are the points $E$ and $B$ of Figure 7) going through five points as a time. Symmetry considerations then show that point $C$ of Figure 7 is a vertex, and similarly for the other points. Thus Figure 8 does describe the vertex distribution of a regular ideal dodecahedron.

It is now a simple matter to explicitly find the equations for planes orthogonal to the fivefold and sixfold axes described above. We seek a circle, centre the origin and of radius $\sigma$, inversion in which interchanges the points 1 and $w$. This requires

$$
\sigma^{2}=w, \quad w^{2}+(2-3 \tau) w+1=0 .
$$

It is now obvious that this plane slices through $\mathcal{D}$ as a pentagon parallel to a face, and that edges of $\mathcal{D}$ are met symmetrically. Since the hypersurface meets each edge orthogonally, the dihedral angle $\pi / 3$ at each edge is also the angle at the vertex of each pentagon in the induced tessellation of this plane. 


\section{YET ANOTHER DESCRIPTION}

Consider again the stereographic projection of the dodecahedron from the north pole viewed as the endpoint of a fivefold symmetry axis. Take any face adjacent to the bottom face, and consider the circle $C_{0}$ at infinity of hyperbolic space passing through the ideal vertices of this face. If we take a family $C_{t}$ of meridianal circles on $S^{2}$, parallel to $C_{0}$, and with $C_{1}$ passing through the vertices of the opposite face of the dodecahedron, for some $t=\tau$ we must have the north pole lying on $C_{\tau}$. Each of these circles projects to a circle in the plane, with $C_{\tau}$ projecting to a straight line. Each of these circles defines an inversion interchanging the same two points, the endpoints of the axis of the dodecahedron orthogonal to the hyperbolic planes corresponding to the circles. The Euclidean centres of the projections of the circles $C_{t}$ describe a straight line on the complex plane, with one point at infinity, whereas the hyperbolic axis about which they are simultaneously symmetric has fixed endpoints. The Euclidean centres of $C_{0}$ and $C_{1}$ can be found as the intersections of the perpendicular bisectors of the edges of the respective pentagons.

Thus an interesting aspect of the projections of the dodecahedron we have given has been brought to light by the family of hyperbolic planes we have been constructing.

The hyperbolic plane parallel to a face, meeting edges orthogonally, determines a reflection of hyperbolic space which interchanges the endpoints of these intersected edges. In the plane, inversion in the corresponding circle interchanges the endpoints of the straight-line segments corresponding to these edges. Accordingly, each of these edges is orthogonal to the circle, so the centre of the circle is the common point of intersection of these edges extended into the pentagon. This is true for each of the pentagons. This leads to both the convenient means of calculating the dihedral angle of two of these hyperbolic planes, given above, and also an alternative way of constructing a stereographic projection of a regular dodecahedron.
Construct an immersed decagon in the plane by taking ten edges of equal length and laying them end to end with interior angles alternately equal to $\pi / 3$ and $7 \pi / 15$. The configuration so obtained is shown in Figure 9, left. The interior of this figure is a nonregular decagon. Construct a regular pentagram with vertices at the vertices of this decagon of smaller angle. The interior pentagon is one of the faces of the dodecahedron. Construct a larger pentagon by extending all edges through the $7 \pi / 15$-vertices, and taking vertices determined by the intersections of such extended edges on either side of a $\pi / 3$ vertex. Construct a pentagram on these vertices. The pentagon interior to this pentagram has 5 edges, with an irregular pentagon based on each edge, in the interior of the pentagon. The construction is illustrated in Figure 9, middle. Finally, Figure 9, right, we delete some extraneous edges, to obtain the projected dodecahedron with edges extended to intersect concurrently in the interiors of faces.

That this configuration really is the regular dodecahedron can be seen by studying the various angles in Figure 9, and comparing with Figure 7.

\section{MATRIX GENERATORS FOR THE TESSELLATION $\{5 ; 6\}$}

That totally geodesic surfaces might exist in the dodecahedral knot complements was suggested in part by the great similarity of their length spectra, calculated by SnapPea [Weeks 1990-96].

The immersed surfaces in the knot complements are covered by hyperbolic planes, tessellated by $\pi / 3$-angled pentagons. The group generated by a fivefold and sixfold rotation, about coplanar disjoint axes, acts transitively on the cells of this tessellation. In the upper half space model, the sixfold rotation with fixed points at $\infty$ and $u$ is determined by the matrix

$$
\left(\begin{array}{cc}
\xi & -i u \\
0 & \xi^{-1}
\end{array}\right)
$$



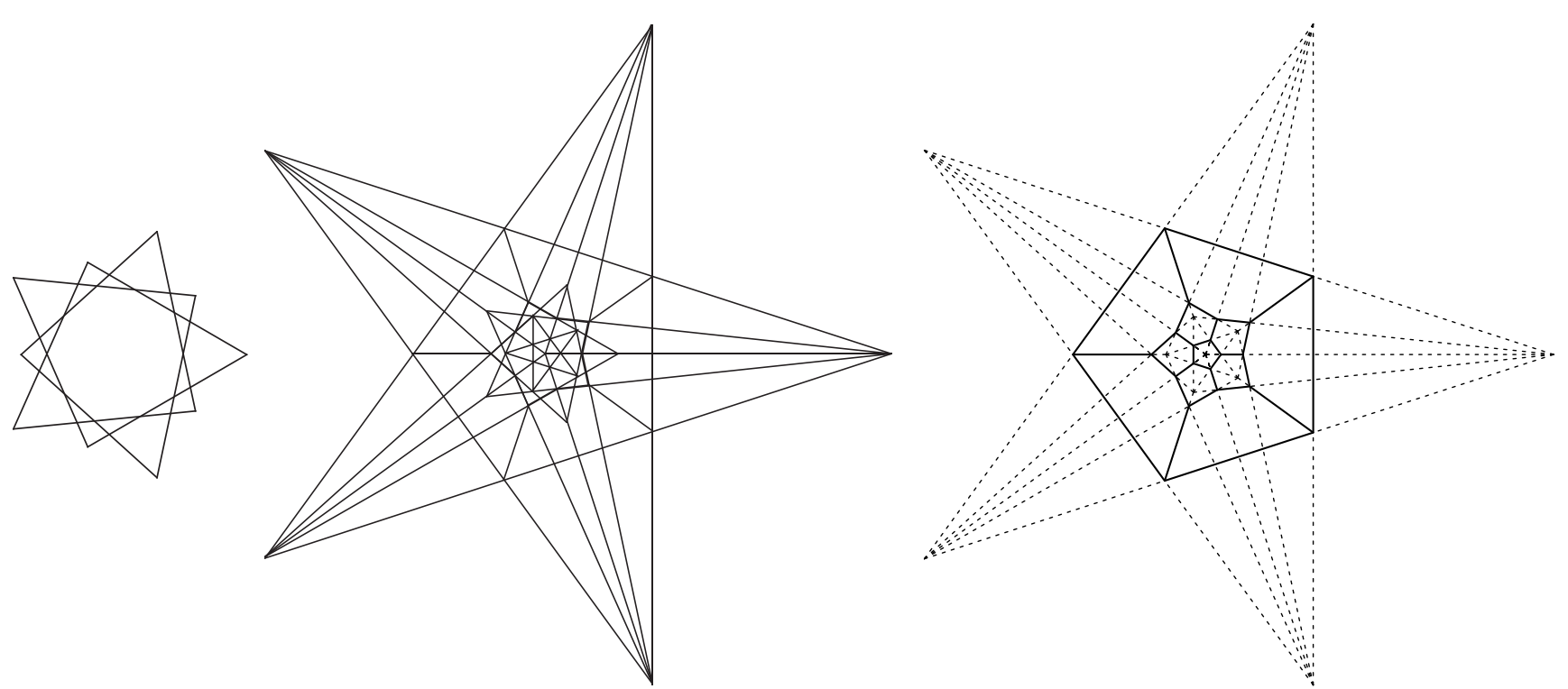

FIGURE 9. Left: An immersed decagon with alternating angles $\pi / 3$ and $7 \pi / 15$. Middle: The extended decagon. Right: Erasing certain lines leads again to the pattern of the ideal regular dodecahedron, as in Figure 8.

For convenience, we may take as generators for the tessellation the two matrices

$$
\left(\begin{array}{cc}
-\tau & -1 \\
1 & 0
\end{array}\right) \text { and }\left(\begin{array}{cc}
\xi & i \tau \xi^{2} \\
0 & \xi^{-1}
\end{array}\right)
$$

The latter defines a rotation with fixed points at $-\tau \xi^{2}=-\tau / 2+\sqrt{3} \tau / 2 i$ and $\infty$.

\section{LENGTH SPECTRA}

The symmetry of the the universal cover of the dodecahedral knots had suggested the possibility of existence of immersed totally geodesic surfaces. This viewpoint was reinforced by the similarity of length spectra for the two knot complements, obtained using SnapPea [Weeks 1990-96]. We have listed these spectra in Table 1, since their intriguing similarity is not yet understood.

It is a remarkable empirical fact that the spectrum for $D_{s}$ appears as a subset of the spectrum of $D_{f}$, at least up to a cutoff of 6.0 with a radius of 7.0. The complex lengths common to both are indicated with "sym". Although only four significant places are given, the agreement is actually to twelve decimal places. The gaps in the table thus correspond to complex lengths occuring for $D_{f}$ but not for $D_{s}$. Note that the first difference between the fibred and symmetric knots occurs for an element with $\pi$ rotational part, with length that of a totally-geodesic surface element.

Proposition 12.1. For both $D_{f}$ and $D_{s}$, the knot complement admits an orientation-reversing involution. Hence all complex lengths occur in conjugate pairs.

Proof. Each knot can be arranged on the graph of a dodecahedron on a two-sphere in $S^{3}$. In each case, the involution interchanges the centres of the ideal dodecahedra of the complement.

For $D_{f}$, take the equator as a Hamiltonian circuit for a nonregular dodecahedron. Twisted bands are added along five arcs in each hemisphere (from which structure we know the knot fibres). The five arcs at the back and front are interchanged by a rotation of the sphere, and a further reflection in the sphere gives the desired involution; the plumbings at the back and front occur with different parity. The shading of the dodecahedron giving rise to $D_{s}$ is the more symmetric. Rotate the sphere by an 


\begin{tabular}{|c|c|c|c|c|c|c|c|c|c|}
\hline 1.9848 & $0.4276^{*}$ & 4.6758 & $0.4505^{*}$ & 5.0821 & $0.4421^{*}$ & 5.2995 & 0.2389 & 5.4649 & 0.0291 \\
\hline 2.7432 & $0.2107^{*}$ & 4.6917 & $0.1444^{*}$ & 5.0910 & $0.0436^{*}$ & 5.3042 & $0.0712^{*}$ & 5.4682 & $0.0655^{*}$ \\
\hline 2.8211 & $0.1184^{*}$ & 4.7136 & $0.0695^{*}$ & 5.0945 & $0.1307^{*}$ & 5.3087 & $0.2959 *$ & 5.4736 & $0.1069^{*}$ \\
\hline 2.9016 & $0.2446^{*}$ & 4.7235 & $0.2239^{*}$ & 5.0982 & $0.3832^{*}$ & 5.3119 & $0.4793^{*}$ & 5.4745 & 0.3454 \\
\hline 3.0687 & $0.0000 *$ & 4.7272 & $0.4645^{*}$ & 5.1039 & $0.2291^{*}$ & 5.3152 & $0.3153^{*}$ & 5.4761 & 0.3056 \\
\hline 3.1284 & $0.4604^{*}$ & 4.7681 & 0.3513 & 5.1115 & $0.3777^{*}$ & 5.3213 & $0.4047^{*}$ & 5.4770 & $0.2696^{*}$ \\
\hline 3.2043 & $0.3442^{*}$ & 4.7836 & $0.0670^{*}$ & 5.1167 & $0.4401^{*}$ & 5.3238 & 0.0952 & 5.4809 & $0.3167^{*}$ \\
\hline 3.4739 & $0.0819^{*}$ & 4.7983 & $0.2951^{*}$ & 5.1195 & 0.1436 & 5.3256 & 0.4572 & 5.4865 & $0.4214^{*}$ \\
\hline 3.5759 & $0.4151^{*}$ & 4.8029 & $0.3106^{*}$ & 5.1393 & 0.3219 & 5.3285 & 0.2917 & 5.4878 & $0.4399^{*}$ \\
\hline 3.6071 & $0.0000 *$ & 4.8113 & $0.2122^{*}$ & 5.1449 & 0.4334 & 5.3312 & $0.2404^{*}$ & 5.4929 & $0.0759^{*}$ \\
\hline 3.6647 & $0.4285^{*}$ & 4.8132 & $0.4966^{*}$ & 5.1729 & 0.3556 & 5.3329 & $0.3775^{*}$ & 5.4935 & 0.1985 \\
\hline 3.7358 & $0.2546^{*}$ & 4.8306 & $0.4575^{*}$ & 5.1754 & $0.1339^{*}$ & 5.3333 & $0.4489^{*}$ & 5.4951 & 0.4976 \\
\hline 3.7854 & $0.2463^{*}$ & 4.8507 & $0.2147^{*}$ & 5.1904 & $0.4718^{*}$ & 5.3374 & 0.3967 & 5.5002 & $0.0000^{*}$ \\
\hline 3.8794 & 0.1349* & 4.8644 & $0.0000^{*}$ & 5.1911 & $0.3808^{*}$ & 5.3405 & $0.4284^{*}$ & 5.5006 & 0.2592 \\
\hline 3.9449 & $0.4647^{*}$ & 4.8659 & $0.2963^{*}$ & 5.1938 & $0.4662^{*}$ & 5.3555 & $0.3274^{*}$ & 5.5038 & $0.3236^{*}$ \\
\hline 3.9451 & $0.4152^{*}$ & 4.8690 & $0.4550^{*}$ & 5.1968 & 0.3222 & 5.3596 & $0.4274^{*}$ & 5.5041 & $0.4696^{*}$ \\
\hline 3.9696 & $0.1446^{*}$ & 4.8704 & $0.4235^{*}$ & 5.2012 & $0.2732^{*}$ & 5.3675 & $0.0000^{*}$ & 5.5048 & $0.3452^{*}$ \\
\hline 4.0869 & $0.4179 *$ & 4.9058 & $0.0000^{*}$ & 5.2083 & $0.0539^{*}$ & 5.3686 & $0.1544^{*}$ & 5.5082 & $0.2483^{*}$ \\
\hline 4.1730 & $0.2691^{*}$ & 4.9058 & $0.2879 *$ & 5.2144 & $0.3425^{*}$ & 5.3818 & $0.0493^{*}$ & 5.5160 & 0.2234 \\
\hline 4.2444 & $0.1706^{*}$ & 4.9080 & 0.0874 & 5.2145 & 0.4430 & 5.3820 & 0.0999* & 5.5222 & 0.0748 \\
\hline 4.2859 & $0.5000^{*}$ & 4.9234 & $0.1020^{*}$ & 5.2153 & 0.1681 & 5.3847 & 0.0610 & 5.5249 & $0.4449^{*}$ \\
\hline 4.3357 & $0.0000^{*}$ & 4.9279 & $0.3353^{*}$ & 5.2182 & $0.1826^{*}$ & 5.3859 & $0.4785^{*}$ & 5.5302 & $0.0000^{*}$ \\
\hline 4.3357 & 0.4000 & 4.9284 & $0.0474^{*}$ & 5.2194 & $0.1446^{*}$ & 5.3932 & $0.2974^{*}$ & 5.5305 & 0.0390 \\
\hline 4.3425 & $0.1179^{*}$ & 4.9505 & 0.1955 & 5.2252 & 0.0000 & 5.3945 & 0.2742 & 5.5322 & 0.0566 \\
\hline 4.4031 & $0.2756^{*}$ & 4.9563 & $0.0613^{*}$ & 5.2334 & 0.4857 & 5.3968 & $0.3716^{*}$ & 5.5359 & $0.4082^{*}$ \\
\hline 4.4374 & $0.2265^{*}$ & 4.9567 & 0.1251 & 5.2452 & $0.1653^{*}$ & 5.4035 & $0.4018^{*}$ & 5.5370 & $0.2496^{*}$ \\
\hline 4.5334 & 0.2791 & 4.9649 & $0.4836^{*}$ & 5.2473 & $0.1796^{*}$ & 5.4120 & 0.2933* & 5.5387 & $0.4862^{*}$ \\
\hline 4.5370 & $0.0468^{*}$ & 4.9709 & $0.0000^{*}$ & 5.2508 & $0.0732^{*}$ & 5.4124 & 0.0983 & 5.5406 & $0.0107^{*}$ \\
\hline 4.5462 & 0.3930* & 4.9877 & $0.4092^{*}$ & 5.2521 & 0.4540 & 5.4161 & 0.1851* & 5.5422 & $0.0347^{*}$ \\
\hline 4.5506 & 0.4776 & 4.9892 & $0.4475^{*}$ & 5.2547 & 0.2949 & 5.4251 & $0.1294 *$ & 5.5436 & 0.3729 \\
\hline 4.5663 & 0.1959* & 5.0083 & 0.2901* & 5.2636 & $0.0323^{*}$ & 5.4257 & $0.0070 *$ & 5.5439 & $0.4189^{*}$ \\
\hline 4.5725 & 0.3189* & 5.0147 & $0.3800^{*}$ & 5.2662 & $0.1193^{*}$ & 5.4308 & $0.2450^{*}$ & 5.5511 & $0.4618^{*}$ \\
\hline 4.5829 & $0.4382^{*}$ & 5.0318 & $0.2852^{*}$ & 5.2690 & 0.3272 & 5.4326 & 0.0481 & 5.5519 & 0.3238 \\
\hline 4.5829 & $0.1533^{*}$ & 5.0344 & $0.1862^{*}$ & 5.2748 & 0.1680 & 5.4340 & $0.0296^{*}$ & 5.5527 & $0.3449^{*}$ \\
\hline 4.5934 & $0.4743^{*}$ & 5.0381 & 0.2628 & 5.2752 & $0.2426^{*}$ & 5.4382 & 0.0182 & 5.5539 & $0.1837^{*}$ \\
\hline 4.6233 & 0.1693* & 5.0478 & $0.4069^{*}$ & 5.2782 & 0.2707 & 5.4389 & 0.4236 & 5.5551 & 0.3686 \\
\hline 4.6250 & 0.1497 & 5.0562 & 0.0582 & 5.2848 & 0.4263 & 5.4408 & $0.2190 *$ & 5.5556 & $0.0803^{*}$ \\
\hline 4.6366 & $0.2008^{*}$ & 5.0753 & $0.3133^{*}$ & 5.2862 & 0.1312 & 5.4423 & $0.1823^{*}$ & 5.5597 & $0.0451^{*}$ \\
\hline 4.6674 & 0.0166 & 5.0782 & $0.3567^{*}$ & 5.2903 & 0.2047 & 5.4606 & $0.0959 *$ & 5.5598 & $0.1960^{*}$ \\
\hline 4.6701 & $0.3257^{*}$ & 5.0796 & $0.2557^{*}$ & 5.2977 & $0.4180^{*}$ & 5.4649 & $0.4367^{*}$ & 5.5599 & 0.0000 \\
\hline
\end{tabular}

TABLE 1. Lengths of closed geodesics for the complements of $D_{f}$ (all entries) and $D_{s}$ (entries marked with an asterisk). Each entry consists of real and imaginary parts; the real part is the translation distance of the corresponding hyperbolic transformation, and the imaginary part gives the torsion in units of $2 \pi$. The imaginary part can be taken with either sign, since, due to the existence of an orientation-reversing involution, all complex lengths occur in conjugate pairs. 
involution interchanging faces of opposite colour, and again compose with a reflection of $S^{3}$ in the two-sphere. This gives the desired involution of $S^{3}-D_{s}$.

If one considers only the real parts, and conjectures the existence of a $\Gamma$-invariant locally finite family of hyperbolic planes in the universal cover, the similarity of length spectra does not seem unreasonable. However, a Fuchsian element has vanishing rotational part, and only a few of the complex lengths have this property. Only some of those in Table 1 arise as multiples of lengths corresponding to the tessellation $\{5,6\}$.

\section{OPEN PROBLEMS}

Problem 13.1. The two subgroups of $\Gamma$ producing these knots are of the same index, and have the same fundamental domain. The spectra are thus both subsets of a common set, but appear to be closer than one might expect. Explain the similarity of length spectra of the dodecahedral knots.

Problem 13.2. The length spectra of the dodecahedral knots contain several complex lengths with vanishing imaginary part which do not correspond to the surface constructed in this paper. Do they arise from some other immersed totally geodesic surface? Note that there are several naturally occuring noncompact immersed surfaces. Moreover, some of the lengths corresponding to potential immersed surfaces occur for $D_{f}$. Does $D_{f}$ contain some other compact surface not arising from the tessellation?

Problem 13.3. Determine whether or not each of the dodecahedral knots contain infinitely many commensurability classes of immersed totally geodesic closed surfaces.

Problem 13.4. By [Long 1987], each of the dodecahedral knot complements has a finite cover containing an embedded totally geodesic surface. Characterize such covers.
Problem 13.5. In [Aitchison and Rubinstein 1992], it is shown that every three-manifold arising by nontrivial Dehn surgery on each of the dodecahedral knots contains a $\pi_{1}$-injective immersed closed surface, satisfying the 4-plane, 1-line condition [Hass and Scott 1992]. Determine which of these are virtually Haken, in light of the preceding problem.

\section{ACKNOWLEDGEMENTS}

We thank Craig Hodgson, Darren Long, Alan Reid, and Bill Thurston for helpful conversations.

\section{REFERENCES}

[Aitchison and Rubinstein 1992] I. R. Aitchison and J. H. Rubinstein, "Combinatorial cubings, cusps, and the dodecahedral knots", pp. 17-26 in Topology 90 (Columbus, OH, 1990), edited by B. Apanasov et al., de Gruyter, Berlin, 1992.

[Coxeter 1948; 1949] H. S. M. Coxeter, Regular Polytopes, Methuen \& Co. Ltd., London, 1948; 1949. Third edition, Dover, New York, 1973.

[Coxeter 1956] H. S. M. Coxeter, "Regular honeycombs in hyperbolic space", pp. 155-169 in Proceedings of the International Congress of Mathematicians (Amsterdam, 1954), vol. III, Erven P. Noordhoff N.V., Groningen, 1956.

[Hass and Scott 1992] J. Hass and P. Scott, "Homotopy equivalence and homeomorphism of 3-manifolds", Topology 31:3 (1992), 493-517.

[Lee 1985a] Y. W. Lee, "Abelian rank of normal torsionfree finite index subgroups of polyhedral groups", Trans. Amer. Math. Soc. 290:2 (1985), 735-745.

[Lee 1985b] Y. W. Lee, "Abelian rank of normal torsion-free finite index subgroups of polyhedral groups", Trans. Amer. Math. Soc. 290:2 (1985), 735745 .

[Long 1987] D. D. Long, "Immersions and embeddings of totally geodesic surfaces", Bull. London Math. Soc. 19:5 (1987), 481-484.

[Maclachlan 1986] C. Maclachlan, "Fuchsian subgroups of the groups $\operatorname{PSL}^{2}\left(O_{d}\right)$ ", pp. 305-311 in Lowdimensional topology and Kleinian groups (Coventry/Durham, 1984), edited by D. B. A. Epstein, 
London Math. Soc. Lecture Note Ser. 112, Cambridge Univ. Press, Cambridge, 1986.

[Maclachlan and Reid 1987] C. Maclachlan and A. W. Reid, "Commensurability classes of arithmetic Kleinian groups and their Fuchsian subgroups", Math. Proc. Cambridge Philos. Soc. 102:2 (1987), 251-257.

[Menasco and Reid 1992] W. Menasco and A. W. Reid, "Totally geodesic surfaces in hyperbolic link complements", pp. 215-226 in Topology 90 (Columbus, OH, 1990), edited by B. Apanasov et al., de Gruyter, Berlin, 1992.

[Murasugi 1963] K. Murasugi, "On a certain subgroup of the group of an alternating link", Amer. J. Math. 85 (1963), 544-550.

[Neumann and Reid 1992] W. D. Neumann and A. W.
Reid, "Arithmetic of hyperbolic manifolds", pp. 273310 in Topology 90 (Columbus, OH, 1990), edited by B. Apanasov et al., de Gruyter, Berlin, 1992.

[Reid 1991a] A. W. Reid, "Arithmeticity of knot complements", J. London Math. Soc. (2) 43:1 (1991), 171-184.

[Reid 1991b] A. W. Reid, "Totally geodesic surfaces in hyperbolic 3-manifolds", Proc. Edinburgh Math. Soc. (2) 34:1 (1991), 77-88.

[Riley 1989] R. F. Riley, "Parabolic representations and symmetries of the knot $932 "$, pp. 297-313 in Computers in geometry and topology (Chicago, 1986), edited by M. C. Tangora, Lecture Notes in Pure and Appl. Math. 114, Dekker, New York, 1989.

[Weeks 1990-96] J. R. Weeks, "SnapPea - (software for the study of hyperbolic three-manifolds)", 199096. See http://www.geom.umn.edu/locate/snappea.

Iain R. Aitchison, University of Melbourne, Department of Mathematics, Parkville, Victoria 3052, Australia (iain@ms.unimelb.edu.au)

J. Hyam Rubinstein, University of Melbourne, Department of Mathematics, Parkville, Victoria 3052, Australia (rubin@ms.unimelb.edu.au)

Received April 17, 1996; accepted September 14, 1996 